\title{
Food-derived serotonergic modulators: effects on mood and cognition
}

\author{
Sjoerd Hulsken ${ }^{1}$, Antje Märtin ${ }^{1}$, M.Hasan Mohajeri ${ }^{2} \dagger$ and Judith Regina Homberg ${ }^{1 *} \dagger$ \\ ${ }^{1}$ Department of Cognitive Neuroscience, Donders Institute for Brain, Cognition and Behaviour, Radboud University Medical \\ Centre, Geert Grooteplein 21 (route 126), 6525 EZ Nijmegen, The Netherlands \\ ${ }^{2}$ DSM Nutritional Products Ltd, RED Human Nutrition and Health, Basel, Switzerland
}

\section{Abstract}

The most frequently described drugs in the treatment of mood disorders are selective serotonin reuptake and monoamine oxidase (MAO) inhibitors, enhancing serotonin levels in the brain. However, side-effects have been reported for these drugs. Because serotonin levels in the brain are dependent on the availability of the food-derived precursor tryptophan, foods such as chicken, soyabeans, cereals, tuna, nuts and bananas may serve as an alternative to improve mood and cognition. Here we discuss the effects of high- or low-tryptophancontaining food, as well as plant extracts with a modest monoamine reuptake and MAO-A inhibition functional profile, on mood and cognition in healthy and vulnerable human subjects and rodents. Together the studies suggest that there is an inverted U-shaped curve for plasma tryptophan levels, with low and too high tryptophan levels impairing cognition, and moderate to high tryptophan levels improving cognition. This relationship is found for both healthy and vulnerable subjects. Whereas this relationship may also exist for mood, the inverted U-shaped curve for plasma tryptophan levels and mood may be based on different tryptophan concentrations in healthy $v$. vulnerable individuals. Animal studies are emerging and allow further understanding of effects and the mode of action of food-derived serotonergic components on mood, cognition and mechanisms. Ultimately, insight into the concentrations of tryptophan and other serotonergic components in food having beneficial effects on mood and cognition in healthy, but particularly vulnerable, subjects may support well-being in our highly demanding society.

\section{Key words: Tryptophan: Monoamine reuptake: Serotonin levels: Food: Emotion: Cognition: Therapy}

\section{Introduction}

Food is a primary requirement to live. Yet, even when food is abundantly available, food could also be used as a powerful tool to increase mental well-being. Stress-related mental disorders such as mood or anxiety disorders are the most prevalent (10 to $25 \%$ women and 5 to $12 \%$ men are being diagnosed each year ${ }^{(1)}$ ), and burdensome, psychiatric disorders. They are characterised by low mood states and cognitive impairments such as reduced learning and memory ${ }^{(1)}$. Thus, the exploitation of resilience or mood/ cognition-enhancing food is of extreme value. Yet, whereas pharmacological manipulations have been abundantly applied, surprisingly little is known about the effects of food on mood and cognition.

The monoamine systems are strongly tied to stress resilience and the (patho)physiology of mood and mood disorders. As such, tricyclic antidepressants (inhibiting serotonin and noradrenaline reuptake) and selective serotonin reuptake inhibitors (SSRI) are frequently prescribed drugs in the treatment of these disorders. To a limited extend these agents also inhibit dopamine reuptake. Whereas antidepressant drugs are considered as healthy drugs, their uncontrolled intake is not without risks. For instance, at a young age these drugs may affect brain development and increase suicide ${ }^{(2)}$. Furthermore, triple monoamine reuptake inhibition corresponds to the function of cocaine, which is a powerful psychostimulant with abuse potential. In addition to monoamine reuptake inhibitors, also drugs that inhibit the breakdown of monoamines are used to improve mood, particularly monoamine oxidase A (MAO-A) inhibitors. However, these agents are also associated with substantial side effects. A major risk is the serotonin syndrome; too high levels of serotonin are toxic. Hence, whereas monoamine reuptake inhibitors and MAO-A inhibitors improve mood, particularly in individuals with a low mood state, their use is not without risks.

\footnotetext{
Abbreviations: 5-HT, 5-hydroxytryptamine; 5-HTT, serotonin transporter; 5-HTTLPR, serotonin-transporter-linked polymorphic region; ATD, acute tryptophan depletion; ATS, acute tryptophan supplementation; LNAA, large neutral amino acid; MAO-A, monoamine oxidase A; SSRI, selective serotonin reuptake inhibitor; TpH2, tryptophan hydroxylase 2; Trp, tryptophan.
}

*Corresponding author: Dr J. R. Homberg, fax +31 2435414 35, email j.homberg@cns.umcn.nl

†Equal contributions to the paper. 
To improve mood by other means, food with high tryptophan (Trp) availability and modest monoamine reuptake and MAO-A inhibition functional profile may be effective. The serotonin precursor $\operatorname{Trp}$ is an essential amino acid that in mammals can only be derived from food $^{(3)}$. Therefore, Trp levels in food appear to be an essential link in the relationship between food intake and our mood state. Accordingly, high-Trp-containing foods (for example, chicken, soyabeans, cereals, tuna, nuts and bananas) are presented on the Internet as food to improve mood. Furthermore, sometimes unknowingly, the Trp content of food has been increased. For instance, Neolithic Mesopotamia-cultivated chickpeas contained more Trp than wild varieties ${ }^{(4)}$. Another example is the treatment of maize with alkali by various cultures in America, which enhances the bioavailability of $\operatorname{Trp}^{(5,6)}$. More recently, research into $\alpha$-lactalbumin has revealed that this whey-derived protein has the highest Trp content of all proteins found in food and therefore is added to infant milk formulas ${ }^{(7)}$. Finally, an even more promising source of Trp is egg protein hydrolysate ${ }^{(8)}$. Pharmacological studies have confirmed that products that increase Trp levels increase serotonin levels in the brain, and apparently modulate processing in the neurocircuits regulating mood to enhance $\operatorname{mood}^{(9,10)}$. Trp depletion, on the other hand, is well known to affect mood negatively ${ }^{(11)}$. Hence, controlling the amount of Trp in food has substantial effects on mood regulation and well-being, although data as discussed in the present review indicate that the relationship between Trp levels and mood is less straightforward than we tend to think. Foods having triple monoamine reuptake and MAO-A inhibitor function have been less extensively investigated, even though they may offer another natural source to boost mood. Indeed, these foods have significant anti-depressive and anxiolytic effects in rats which are comparable with the effects of antidepressant reference drugs ${ }^{(12)}$

The aim of the present review is to provide an update about foods influencing the serotonergic system that have potential to increase stress resilience and thereby our well-being. Rather than applying pharmacological drugs when pathology has developed, prevention by healthy foods may decrease the burden associated with mood disorders. An important consideration is the heterogeneity of mood disorders, caused by genetic or other biological factors. Along with the fact that there are large individual differences in vulnerability to mood disorders, there are large individual differences in responsivity to antidepressant agents ${ }^{(13)}$, as well as in responsivity to serotonergic food components. These individual differences play a leading role in our discussion of the effects of serotonergic foods on mood. In sum, the present review discusses the serotonergic system, and the influence of serotonergic food components on mood and cognition in healthy, genetically vulnerable and clinically depressed and recovered populations, as well as (genetic) rodent models.

\section{The serotonergic system}

Serotonin, or 5-hydroxytryptamine $(5-\mathrm{HT})$, is an ancient monoamine, as even some unicellular organisms are able to synthesise it. Furthermore, it is found in basically all animal species and various plants such as stinging nettles and walnuts. Apart from its roles in the cardiovascular and digestive systems, 5-HT is an important neurotransmitter with a wide variety of actions in different brain regions. Because 5-HT as a hydrophilic molecule is unable to cross the blood-brain barrier, it is synthesised in the brain, specifically the serotonergic neurons in the raphe nuclei itself, by the enzyme tryptophan hydroxylase $2(\mathrm{TpH} 2)$. $\mathrm{TpH} 2$ is also expressed in myenteric neurons in the gut ${ }^{(14)}$. TpH2 is distinct from tryptophan hydroxylase 1 (Tph1), the enzyme that is responsible for the 5-HT system in the peripheral system, mainly the gut. The synthesis of 5-HT in the brain takes place in the axon terminals of serotonergic neurons where $\mathrm{TpH} 2$ exerts the first rate-limiting step. TpH2 hydroxylates L-tryptophan and forms 5-hydroxytryptophan. This molecule is subsequently decarboxylated by aromatic L-amino acid decarboxylase to form 5-HT. The 5-HT molecules are stored in vesicles in the serotonergic neurons. Upon an action potential, 5-HT is released from axon terminals of serotonergic neurons into the synaptic cleft where it binds to its receptors on the postsynaptic neuron. Its activity is terminated by the serotonin transporter (5-HTT) in the membrane of the presynaptic neuron that transports $5-\mathrm{HT}$ back into the cell. This allows recycling of 5-HT, although stored/released 5-HT is also degraded by MAO-A. Finetuning of the postsynaptic response to the release of 5-HT is achieved through a host of different receptors and cells and/or brain area-specific receptor profiles, thereby facilitating the different roles that 5-HT plays in physiological and behavioural functions.

Serotonergic cell bodies are located in the raphe nuclei, a cluster of nuclei in the brain stem projecting to almost all brain regions ${ }^{(15)}$, including lateral cortical regions, the amygdala and the hypothalamus, which are all involved in processing emotions and stress. Because TpH2, 5-HTT and MAO-A are expressed in these and other brain areas ${ }^{(16-18)}$, agents acting through TpH2, 5-HTT and MAO-A will have a global effect on 5-HT neurotransmission.

Although the precise role of 5-HT in mood disorders such as depression, obsessive-compulsive disorder and anxiety is not fully understood, it is evident that the 5-HT system is perturbed in these disorders. The synthesis, release, reuptake and/or metabolism of $5-\mathrm{HT}^{(19,20)}$, as well as the functionality of the $5-\mathrm{HT}_{1 \mathrm{~A}}$ and $5-\mathrm{HT}_{2}$ serotonin receptors ${ }^{(21,22)}$, are to different extents affected in anxiety and depression. The general view is that there is an inverse relationship between depression and 5-HT levels, based on the antidepressive properties of serotonin-enhancing drugs ${ }^{(23)}$. In terms of food, a positive association between the consumption of maize, which contains less Trp compared with other food staples, and homicide rates has been found ${ }^{(24)}$. Moreover, 
another cross-national study revealed a negative correlation between dietary $\operatorname{Trp}$ and suicide rates ${ }^{(25)}$.

There is also evidence that genetic variation in the serotonergic system increases vulnerability to anxiety- and depression-related disorders ${ }^{(26-29)}$. A common polymorphism in the promoter region of the 5-HTT (SLC6A4) gene (abbreviated: 5-HTTLPR; serotonin-transporter-linked polymorphic region) is associated with trait anxiety ${ }^{(30)}$, and in the context of (early-life) stress, this polymorphism increases risk for depression ${ }^{(31)}$. Paradoxically, it is the low-activity short (s) allelic variant that increases vulnerability. Although human studies could not provide evidence, studies employing 5-HTT knockout mice and rats have revealed that reduced 5-HTT expression is associated with increased synaptic 5 -HT levels ${ }^{(32)}$. Hence, whereas SSRI-induced increases in 5-HT levels reduce depressive symptoms, genetically induced constitutive high 5-HT levels appear to increase these symptoms. Furthermore, whereas 5-HTTLPR s-allele carriers are at risk for depression if exposed to stress, those that are depressed suffer from decreased responsivity to SSRI. Intuitively, decreased 5-HTT expression leaves a smaller window of opportunity for SSRI to exert their maximum effects.

In 1998, gene mapping of the X-chromosomal MAO-A gene revealed a functional polymorphism in the upstream promoter region, consisting of a variable number of tandem repeats ( $\mathrm{u}-\mathrm{VNTR})$. The different lengths of these variants was found to influence protein transcription and thus enzymic activity ${ }^{(33)}$. Others linked the alleles which confer high enzymic activity (three, four and five repeats) to several anxiety- and depression-related disorders ${ }^{(34-37)}$, as well as reduced responsiveness to antidepressant treatment $^{(37,38)}$.

Finally a number of different TpH2 SNP have been reported in several ethnical cohorts which are thought to cause, through either lower enzymic activity or loss of function, lower extracellular 5-HT levels. Zhang et al. ${ }^{(39)}$ showed that an $80 \%$ loss of function TpH2 G1463A SNP found in a geriatric sample in North Carolina (USA) was more prevalent in the cohort diagnosed with unipolar depression compared with the matching control cohort. Another study revealed in Taiwanese Han Chinese subjects a possible association between bipolar disorder and $\mathrm{TpH} 2$ C2755A, which leads to a reduction of 5-HT synthesis when expressed in cells ${ }^{(40)}$, and $\mathrm{TpH} 2$ Pro206Ser (conferring a $30 \%$ reduction in enzymic activity) was found to be significantly higher expressed in a cohort of German patients suffering from bipolar disorder compared with controls ${ }^{(41)}$. In addition to these SNP, the study on Taiwanese Han Chinese also reported that the $-703 \mathrm{G} /-473 \mathrm{~A}$ haplotype (a $\mathrm{TpH} 2$ promoter variant associated with bipolar disorder) resulted in reduced transcription of the $\mathrm{TpH} 2$ gene as well as reduced affinity for the transcription factor POU3F2 ${ }^{(40)}$.

Because 5-HTT, Thp2 and/or MAO-A polymorphisms affect the functioning of the serotonergic system, it is to be expected that they influence the effects of serotonergic food components, either through reduced capacity to convert Trp into 5-HT (due to reduced $\mathrm{TpH} 2$ function), reduced sensitivity of the serotonergic system to elevated 5-HT levels (due to reduced 5-HTT function) or enhanced degradation of 5-HT (due to elevated MAO-A activity).

\section{Tryptophan}

Since 5-HT cannot cross the blood-brain barrier due to its hydrophilic properties the synthesis of the neurotransmitter takes place in the axon terminals of serotonergic neurons. The precursor Trp is absorbed from food into the bloodstream. When a meal containing protein is consumed a large proportion of Trp entering the portal vein is metabolised in the liver, which does not happen with the other large neutral amino acids (LNAA) ${ }^{(42)}$. Thus the amount of Trp entering the circulation is much less compared with other LNAA, if the amounts consumed are the same. However, the secretion of insulin in response to the intake of carbohydrates in the meal reduces the plasma concentrations of all LNAA except Trp, which binds to albumin and thereby can circulate throughout the body ${ }^{(43,44)}$. Whether a meal increases the ratio of plasma Trp levels compared with the plasma levels of other LNAA (the TRP:LNAA ratio) depends on the amount of protein digested and the release of insulin ${ }^{(45,46)}$. When Trp in the circulation reaches the brain capillaries it is transported across the blood-brain barrier. Here Trp has to compete with other LNAA for active transport across the cell membranes of the epithelial cells lining the capillaries. Therefore, not the plasma level of Trp alone, but the TRP:LNAA ratio determines its rate of transport across the blood-brain barrier ${ }^{(45)}$. Once inside the brain, Trp is taken up by the serotonergic cells of the raphe nuclei and via two enzymic reactions it is converted into 5-HT (see the section on the serotonergic system).

\section{Acute tryptophan depletion}

Since there is a direct relationship between dietary Trp and brain 5-HT synthesis, the supply of 5-HT in the brain can be exhausted by decreasing the availability of Trp. A standardised procedure to drastically decrease the levels of 5-HT is acute tryptophan depletion (ATD). In short, a diet low in Trp is eaten by the subjects for $24 \mathrm{~h}$, after which they fast overnight and during the test day except for a shake which contains all amino acids excluding Trp. This in itself causes a drop in the plasma TRP:LNAA ratio. Furthermore, protein synthesis is stimulated by the intake of high-protein foods or drinks. A protein-rich low-carbohydrate diet results in a decreased amount of Trp in the brain since the TRP:LNAA ratio in normal protein is very low. Therefore, a protein-rich low-carbohydrate diet results in more competition for the transport system over the blood-brain barrier and consequently less transport of Trp into the brain. As a consequence, Trp needed for 
protein synthesis during the diet and test day must be supplied by the reserves in the body. Inevitably these reserves will be drained to the point that they cannot maintain plasma Trp levels, causing the TRP:LNAA ratio to drop even further, to about $15 \%$ within $5 \cdot 5 \mathrm{~h}$. Brain Trp levels drop to $5 \%$ several hours later, which hampers brain 5-HT synthesis ${ }^{(47-49)}$. The effects of ATD in healthy volunteers and vulnerable subjects are described in the following sections.

\section{Effects of acute tryptophan depletion on mood and cognitive functions in healthy subjects}

Mood. In line with the increased incidence of depression in women, it has been reported that the mood of healthy female volunteers but not of healthy male volunteers dropped after ATD (about 80-90\% reduction in plasma Trp $)^{(50,51)}$. In male subjects, effects of ATD on mood were either small ${ }^{(52,53)}$ or fully absent (about 70-90\% reduction in plasma $\operatorname{Trp})^{(50,51,54,55)}$. positron emission tomography (PET) imaging showed that there may be differences in 5-HT synthesis and metabolism between the sexes, which could explain the difference in response to ATD $^{(48)}$. Other studies reported that the performance of men in the Stroop Color Word Test, testing selective attention and response inhibition, improved following ATD (about $60-80 \%$ reduction in plasma Trp) ${ }^{(56,57)}$. However, the effects of ATD on recognition of emotional faces are inconsistent; some observed a decrease in recognition of facial emotions following ATD (no complete data on plasma Trp) in women ${ }^{(58)}$, others found an increase in the recognition of happy faces by women (plasma Trp reduced by $55 \%)^{(59)}$, and still others reported no effects in both men and women following a Trp reduction of $65 \%{ }^{(60,61)}$. Thus, whereas ATD impairs mood in women, there are conflicting results concerning the effects of ATD on the recognition of emotional faces.

Cognition. Apart from mood, ATD has been found to affect a number of cognitive functions in healthy individuals. Memory consolidation was impaired when the learning of a list of words took place during the Trpdepleted state (about 60-80\% reduction in plasma Trp), whereas the recall of word lists learned before the ATD procedure was not affected ${ }^{(56,62)}$. Moreover, McAllister et $a{ }^{(63)}$ showed that ATD leading to a plasma Trp reduction of $85 \%$ significantly impaired source memory recall. In sum, research consistently shows memory impairment following ATD.

\section{Effects of acute tryptophan depletion on mood and cognitive functions in vulnerable subjects}

Mood. In vulnerable and subclinical subjects more consistent evidence has been found pointing towards a lowering of mood and alterations in cognitive functioning in response to ATD. For instance, healthy subjects with a multigenerational family history of affective disorders showed a reduction in mood as measured the by the Profile of Mood States scale following ATD (about 70-90\% reduction in plasma $\operatorname{Trp})^{(64,65)}$. The same is true for patients who are remitted from a depressive episode and responded to SSRI treatment (Trp plasma reduced by about $50-90 \%)^{(66-69)}$; however, see Leyton et al. ${ }^{(70)}$. In remitted depressed patients, ATD impaired attention to positive stimuli (about 55-64\% reduction in plasma $\operatorname{Trp})^{(71)}$ and decreased the recognition of fearful and happy faces (plasma Trp reduced by 55-84\%) ${ }^{(59,72)}$. Furthermore, in otherwise healthy women as well as remitted major depression patients carrying the 5-HTTLPR ss or 1s genotype it was found that ATD significantly lowered mood compared with l-allele carriers (about 70-85\% reduction in plasma Trp) ${ }^{(73-75)}$. Interestingly, men carrying the same genotype did not experience a lower mood, but instead showed increased impulsivity after ATD (plasma Trp reduced by about $75-85 \%)^{(75,76)}$. When ATD $(77 \%$ reduction in plasma Trp) was combined with a stressor, mood deteriorated even further in 5-HTTLPR ss subjects, while no further reduction was found in individuals homozygous for the 1 allele ${ }^{(77)}$. In summary, a vulnerable serotonergic system, whether due to genetic background or a (family) history of affective disorders, seems to enhance the mood-deteriorating effects of ATD.

Cognition. Subclinical subjects tend to suffer from instable cognitive function, which increases the risk of cognitive impairments when cognitive capacities are too low to properly deal with environmental challenges, like stress, but also unhealthy food. In their pooled analysis of nine separate ATD trials, Sambeth et al. ${ }^{(78)}$ discovered that the memory impairment effect of ATD (63-97\% reduction in plasma Trp) was more prominent in women than in men ${ }^{(78)}$. ATD (plasma Trp reduced by 69\%) also lowered incentive motivation in 5-HTTLPR s-allele carriers ${ }^{(79)}$. In remitted depressed patients, ATD impaired immediate verbal recall as well as immediate and delayed recall of lists of spoken words (plasma Trp reduced by $55-71 \%)^{(59,80,81)}$. However, it improved attention to neutral stimuli (about 55-64\% reduction in plasma Trp) ${ }^{(71)}$.

\section{Effects of acute and (sub-)chronic tryptophan depletion in animal models}

While the behavioural effects of ATD on human subjects are well documented, research on the effects of Trp depletion in animal models can offer further insight into the role of 5 -HT in mood and cognitive processes and enable researchers to examine the effects of (sub-)chronic Trp depletion. For instance, sub-chronic Trp depletion (SCTD) (57\% reduction in plasma Trp, prefrontal cortex 5-HT levels reduced by 34\%) in Sprague-Dawley rats led to depression-related behaviour in the forced swim test as well as an increase in corticosterone, aldosterone, 5-HT receptor density and increased N-methyl-D-aspartate 
(NMDA) signalling ${ }^{(82)}$. In another study reward learning was impaired without an effect on motivation, assessed by effort discounting and reversal learning tasks, following SCTD (no data on plasma Trp levels, ventromedial frontal cortex 5-HT levels reduced to about $50 \%$ of control) in Long Evans rats ${ }^{(83)}$. SCTD (no data on plasma Trp levels, prefrontal cortex 5-HT levels reduced to $39 \%$ of control) in Wistar rats also resulted in enhanced proneness to gamble and poor decision making in the rodent Iowa Gambling Task ${ }^{(84)}$. Interestingly, also in macaques performing a gambling task, ATD (about 65\% reduction in plasma Trp) increased risky decision making ${ }^{(85)}$. Chronic Trp depletion in Lister hooded rats $(40-50 \%$ reduction of 5-HT levels in the hippocampus, frontal cortex and striatum) impaired object recognition in the novel objectrecognition paradigm, which was reversed by administering the atypical antipsychotic risperidone ${ }^{(86)}$. ATD studies in rats have also revealed that the effects of ATD (about $50 \%$ reduction in plasma Trp) on hippocampal and frontal cortex 5-HT levels were more pronounced in homozygous serotonin transporter knockout Wistar rats compared with heterozygous knockout rats and wild-type controls ${ }^{(87)}$. Finally, the circadian rhythm of locomotor activity as well as the sleep-wake cycle were found to be disturbed in Sprague-Dawley rats by ATD $(99 \%$ reduction in plasma Trp, $85 \%$ decrease in 5-HT brain levels) ${ }^{(88)}$. In conclusion, animal studies have provided evidence that Trp depletion indeed lowers 5-HT levels in brain areas that are involved in several cognitive functions and mood, thus reinforcing the conclusions drawn from human ATD studies and providing further insight into the underlying processes. However, some care should be taken when reviewing these studies on Trp depletion in rats, as not all strains respond in the same manner to Trp depletion ${ }^{(89)}$.

\section{Summary of acute tryptophan depletion}

In healthy subjects the negative effects of ATD on memory are the most profound, while it seems to enhance selective attention and inhibition of response. Interestingly, the lowering of mood by ATD in healthy subjects is only consistently found in females. This sex effect may be a consequence of lower 5-HT synthesis in females than in males ${ }^{(48)}$, making females more vulnerable to manipulation of the serotonergic system. This is also true for subjects that have a vulnerable serotonergic system as a result of genetic or (family) history of affective disorder, as is seen in the enhanced lowering of mood and negative effects on several cognitive functions in these vulnerable subpopulations.

\section{Acute tryptophan supplementation}

Whereas consumption of a high-protein low-carbohydrate diet lowers Trp levels ${ }^{(90)}$, consumption of a diet high in carbohydrates increases the Trp:LNNA ratio in blood and the brain ${ }^{(44,91)}$. The intake of a high amount of carbohydrates triggers the release of insulin that in turn stimulates the uptake of LNAA into muscle tissue, with the exception of Trp, which is loosely bound by free albumin. Combined with a low intake of protein, the plasma TRP:LNAA ratio shifts in favour of Trp and therefore increases the transport of Trp over the blood-brain barrier. This method, however, only mildly elevates Trp levels in the brain, rarely exceeding an elevation of $25 \%$ over baseline. Furthermore, most typical diets high in carbohydrates are high in protein as well, negating their effect on plasma Trp levels ${ }^{(92)}$. A valid alternative is the administration of pure Trp via intravenous injection in which the precise elevation of pure Trp levels can be observed. This method, however, proves to be quite invasive and inconvenient as a regular event. Yet another approach is oral administration of a drink high in $\alpha$-lactalbumin retrieved from whey which is high in $\operatorname{Trp}^{(7)}$ and therefore can increase plasma Trp without increasing the amount of competing LNAA. In several studies, $\alpha$-lactalbumin was administered as a chocolate-flavoured drink; thus this method is much more feasible for a continued administration and even rather pleasant for the test subject, although the increase in Trp via this method is limited and significantly lower than via intravenous injection ${ }^{(93,94)}$. More recently hydrolysed protein and egg protein hydrolysate in the form of a sweetened drink have been found to raise brain Trp up to $255 \%$, so oral administration does not necessarily produce a smaller increase in plasma Trp than pure intravenous injections ${ }^{(8,9)}$. Independent of the method, an increased amount of Trp in the brain results directly in an increase in 5-HT synthesis since the ratelimiting enzyme $\mathrm{TpH} 2$ is only half saturated under normal circumstances and 5-HTP aromatic L-amino acid decarboxylase is a highly active enzyme ${ }^{(9)}$.

In the last decades, there have been many studies on the effects of acute Trp supplementation (ATS) on mood and cognition, utilising either amino acid drinks containing pure Trp, $\alpha$-lactalbumin, hydrolysed protein or egg protein hydrolysate. Because there is no standardised protocol for ATS, the obtained Trp:LNAA levels after digestion of the ATS mixture or diet can differ greatly between studies. Nonetheless, significant effects of ATS have been observed in both healthy and (sub)clinical populations. In the next sections modulation of various cognitive functions and mood by ATS in healthy and vulnerable subjects will be discussed.

\section{Effects of acute tryptophan supplementation on mood and cognitive functions in healthy subjects}

Mood. ATS seems to modulate emotional processing in healthy subjects, in a dose-dependent manner. An oral dose of $1.8 \mathrm{~g}$ Trp (600\% increase in TRP:LNAA) increased the recognition of fearful and happy faces in females ${ }^{(95)}$. Murphy et al. ${ }^{(96)}$ found increased recognition of happy facial expressions, and a decreased recognition of 
expressions of disgust after $14 \mathrm{~d}$ of $3 \mathrm{~g} \mathrm{Trp} / \mathrm{d}$ (no data on TRP:LNAA ratio) in healthy female subjects. In another study, drinks containing a hydrolysed egg-white protein, which increases plasma TRP:LNAA by 191-255\%, improved $\operatorname{mood}^{(9)}$. However, carbohydrate-rich proteinpoor diets or drinks containing $\alpha$-lactalbumin that increase plasma TRP:LNAA by $21-67 \%$ did not have an effect on $\operatorname{mood}^{(9,93,97-100)}$. Furthermore, unphysiologically high increases in TRP:LNAA ratios negatively affected mood, as was reported after an intravenous injection of $7 \mathrm{~g}$ pure $\operatorname{Trp}\left(1500 \%\right.$ increase in TRP:LNAA) ${ }^{(101,102)}$. Thus, there may be an inverted U-shaped relationship between TRP:LNAA ratios and mood in healthy subjects.

Cognition. Considering that memory consolidation and recall are impaired after ATD and in depression ${ }^{56,62,63)}$, ATS may enhance these cognitive functions. Indeed, it has been reported that an increase of $21 \%$ in TRP:LNAA following the administration of two $\alpha$-lactalbumin-rich drinks improved abstract visual memory in healthy subjects ${ }^{(100)}$. However, Sobczak et al. ${ }^{(102)}$ found that an intravenous injection of $7 \mathrm{~g}$ Trp had an adverse effect on performance in memory tests. Given that extreme elevation of TRP:LNAA (1500\%) caused significant sedation in the test subjects ${ }^{(102,103)}$, these detrimental effects could well be due to sedation and not a direct effect of increased 5-HT levels. For instance, ATS (770\% increase in plasma free Trp, no data on TRP:LNAA ratio) has been found to increase reaction times ${ }^{(96,104)}$ and subjective fatigue ${ }^{(105)}$ in both healthy subpopulations. Thus, ATS-induced TRP:LNAA increases of $21-43 \%$ have beneficial effects on memory functions, whereas higher increases impair memory, potentially due to sedative effects.

\section{Effects of acute tryptophan supplementation on mood and cognitive functions in vulnerable subjects}

Mood. In contrast to ATS effects on mood in healthy subjects, an increase in the plasma TRP:LNAA ratio of $41-48 \%$ following ATS was sufficient to improve mood as well as vigor in high-stress-vulnerable subjects undergoing laboratory stress ${ }^{(93,97)}$. Other studies found that in women with premenstrual complaints, ATS (29\% increase in Trp:LNAA) improved mood during the premenstrual stage ${ }^{(106,107)}$. Furthermore, in 5-HTTLPR s-allele, but not 1-allele, carriers, ATS (increase in plasma TRP:LNAA of 190\%) was found to have positive effects on mood and vigor after exposure to laboratory stress ${ }^{(108)}$. It has also been reported that ATS (no data on TRP:LNAA levels) was without effect on mood in 5-HTTLPR s- and 1-allele carriers, but lowered hypothalamic-pituitary-adrenal axis activity in individuals being homozygous for the s allele ${ }^{(109)}$. In addition, ATS reduced food intake in dieting females regardless of their 5-HTTLPR genotype, while increasing mood and emotional eating solely in 1/1 subjects (plasma TRP:LNAA ratio increased by $70 \%)^{(110)}$. However, it has also been demonstrated that an $\alpha$-lactalbumin-enhanced diet (21\% increase in TRP:LNAA) failed to modulate mood or vigor in recovered depressed subjects ${ }^{(98,100)}$, and an increase of $21 \%$ in TRP:LNAA following the administration of two $\alpha$-lactalbumin-rich drinks had no effect on mood in recovered depressed patients ${ }^{(100)}$. Perhaps these TRP:LNAA ratios of about $20 \%$ are too low to improve mood in vulnerable subjects. Overall, ATS seems to have more profound effects on mood in vulnerable populations than in healthy subjects. Potentially, factors such as lower baseline levels of 5-HT and reduced capacity to clear released 5-HT from the synaptic cleft may help to clarify whether ATS has beneficial effects in individuals with mood disturbances.

Cognition. An increase of $21 \%$ in TRP:LNAA following the administration of two $\alpha$-lactalbumin-rich drinks improved abstract visual memory in both healthy controls and recovered depressed patients ${ }^{(100)}$. Another study found that in women with premenstrual complaints, ATS improved memory for abstract figures (29\% increase in TRP:LNAA) and word recognition (6-25\% increase in TRP:LNAA) during the premenstrual stage ${ }^{(106,107)}$. Furthermore, Markus et al. ${ }^{(111)}$ reported an enhanced short-term memory scanning in healthy high-stress-prone subjects following laboratory stress and after consumption of a high-carbohydrate low-protein diet (TRP:LNAA ratio increased by $42 \%$ ) or after consumption of a diet enriched with $\alpha$-lactalbumin (Trp:LNAA ratio increased by $43 \%)^{(112)}$. Together these data suggest that also in vulnerable subjects ATS improves memory-related processes, at rather low increases in plasma TRP:LNAA ratios. As to whether the high Trp-induced sedative effects on cognition as observed in healthy subjects also take place in vulnerable subjects needs to be investigated.

\section{Effects of acute and (sub-)chronic tryptophan supplementation in animal models}

As in the case of Trp depletion, the added value of studying Trp supplementation in animals lies in the ability to provide direct 5-HT level read-outs from the whole brain or specific brain areas and assess (sub-)chronic effects of Trp supplementation. Feurté et al. ${ }^{(113)}$ showed that chronic supplementation of the diet of rats with $\alpha$-lactalbumin leads to a stable increase of $40 \%$ in plasma TRP:LNAA. Increasing plasma TRP:LNAA levels in rats were found to increase brain 5-HT levels, as dietary sub-chronic Trp supplementation (30\% increase in brain TRP:LNAA) in Sprague-Dawley rats resulted in an increase of $92 \%$ in extracellular 5-HT levels in the dorsal hippocampus. Moreover, the effect of the 5-HT-releasing agent fenfluramine was enhanced as was performance in a differential reinforcement of low rate responding condition (mimicking SSRI treatment) ${ }^{(114)}$. Collins et al. ${ }^{(115)}$ showed that supplementation of an egg white protein hydrolysate replenished brain serotonin stores, even after pharmacological depletion of serotonin from the brain by 3,4-methylenedioxymethamphetamine ${ }^{(115)}$. In Wistar rats chronic 
treatment with $\operatorname{Trp}$ by oral administration (about 60\% increase in plasma Trp:LNAA) was shown to increase 5-HT levels in the hippocampus by $57 \%$, in the cortex by $46 \%$ and by $72 \%$ in the rest of the brain, as well as boosting performance in the radial arm maze test and water maze test, indicating improvement in both short- and long-term memory ${ }^{(116,117)}$. However, a $7 \mathrm{~d}$ Trp treatment in mice suppressed food intake significantly and, more worryingly, increased brain thiobarbituric acid-reactive substances (a measure for oxidative stress) ${ }^{(118)}$. Taken together, these data show that it might be worthwhile to investigate whether the positive effects of ATS in human subjects could be maintained when diet is supplemented with Trp over a longer period of time. Although some care should be taken, the increase in oxidative stress following chronic Trp supplementation in mice should not be taken lightly.

\section{Summary of acute tryptophan supplementation}

ATS seems to improve mood and cognition in both healthy and vulnerable subjects. Effects of ATS on cognition are more consistent and require lower increases in the plasma TRP:LNAA ratios than needed to improve mood. Yet, for both mood and cognition it has been shown that too high increases in Trp levels result in poorer performances. As serotonin synthesis can theoretically only be increased to a maximum of $200 \%$ due to saturation of $\mathrm{TpH} 2$ (see the acute tryptophan supplementation section), it is not likely that the poor performance results from local negative responses to elevated serotonin levels like sensitisation. However, a possible explanation could be sedation, which was reported in one study ${ }^{(105)}$, caused by the subsequent synthesis of melatonin from serotonin by serotonin- $N$-acetyltransferase and hydroxyindole- $O$ methyltransferase ${ }^{(119)}$. Taken together, these data suggest that there is an inverted U-shaped curve for plasma Trp levels and mood/cognition. Finally, research in animal models has provided results that seem to justify further investigation of chronic Trp supplementation in human subjects.

\section{Triple monoamine inhibitors}

Unbeknownst to the individuals of ancient and medieval times, some of the medicinal herbs they used actually contained monoamine reuptake transporter inhibitors. During the previous decade a number of studies focused on the antidepressant-like effects of extracts from several subspecies of St John's wort (Hypericum perfoliatum), which was traditionally used to treat wounds, eczema and disorders of the central nervous system ${ }^{(120)}$. Not only did these studies reveal replicable antidepressant effects in mice ${ }^{(121-124)}$, analysis of monoamine uptake by rat synaptosomes showed that uptake of all three monoamines was inhibited by these extracts ${ }^{(121,124)}$. In addition to St John's wort, the presence of monoamine reuptake inhibitors was shown in the extracts of Siphocampylus verticillatus (a Brazilian medicinal plant $)^{(125)}$, in Fructus Akebiae (a traditional Chinese cure for depressive disorders) ${ }^{(126)}$ and Sideritis scardica (used as a tea, flavouring agent and universal remedy around the Mediterranean) ${ }^{(127)}$.

Perhaps even more surprising is the presence of monoamine transporter inhibitors in regular foods. In 2003, a group of researchers reported on a group of isoflavans and isoflavenes, which are present in licorice, with serotonin transporter (SERT)-inhibiting properties ${ }^{(128)}$. After $7 \mathrm{~d}$ of oral injections with the extract of the roots of Glycyrrbiza glabra (from which licorice is made), mice showed enhanced learning and memory performance in the passive avoidance paradigm and reduced anxiety in the elevated plus maze ${ }^{(129)}$, as well as reduced immobility in the forced swim and tail suspension tests for depression ${ }^{(130)}$. These findings suggest that the extract from G. glabra possesses antidepressant potentials. Recently, Mechan et al. ${ }^{(12)}$ demonstrated that a supercritical $\mathrm{CO}_{2}$ oregano extract acting as a moderate noradrenaline, serotonin and dopamine reuptake inhibitor and MAO inhibitor exerted antidepressant-like and anxiolytic effects in mice. In the same study, the researchers used in vivo microdialysis in rats to confirm that extracellular 5-HT levels were indeed higher after a dose of the oregano extract, albeit more slightly compared with the antidepressant fluoxetine. Moreover, electroencephalogram measurements after acute oral supplementation in freely moving rats showed that this extract may exhibit antidepressant and neuroprotective activities ${ }^{(131)}$. Taken together, the results of these studies seem to justify further investigation into possible other dietary sources of monoamine reuptake inhibitors, as well as studies testing whether the beneficial behavioural effects of these plant extracts found in mice can be replicated in human subjects. An important fact to note is that the half maximal inhibitory concentration $\left(\mathrm{IC}_{50} \mathrm{~s}\right)$ of these plant-derived monoamine reuptake inhibitors are about 1000-fold lower than those of pharmacological compounds, making the chances of detrimental side effects much lower ${ }^{(12)}$. In addition, a recent study investigating the attitudes of the general public and general practitioners towards enhancement of mood and cognition in healthy individuals by either pharmaceuticals or natural compounds showed that the public, especially, tends to be more favourable towards natural compounds ${ }^{(132)}$.

\section{Summary and conclusion}

While the serotonergic system is an important target for many pharmacological agents applied in psychiatry, this system is also amenable to influences of food. Indeed, food containing a low TRP:LNAA ratio tends to reduce mood and cognition, and food that increases this ratio may increase mood and cognition in humans. However, as we reviewed, the relationship between Trp intake and 
behavioural performance is not unequivocal. In part this is because methods that have been used to lower or increase the plasma TRP:LNAA ratio differ considerably across studies. Nonetheless, there may be an inverted U-shaped relationship between central 5-HT levels and optimal mood/cognition; low and too high Trp levels impair $\mathrm{mood} /$ cognition, and moderate to high Trp levels improve mood/cognition. Regarding cognition this relationship is found for both healthy and vulnerable subjects (Fig. 1(a)). However, regarding mood, effects of ATD and ATS seem to depend on the functioning of the serotonergic system of vulnerable or subclinical subjects, with a much more profound decrease in mood of vulnerable subjects compared with healthy subjects in the lower brain Trp range (Fig. 1(b)). However, if brain Trp levels are in the optimum range, mood in vulnerable subjects is comparable with mood in healthy subjects. In healthy individuals an increase in plasma TRP:LNAA of $67 \%$ does not modulate mood, while in vulnerable subjects an increase of $20-50 \%$ is sufficient to normalise their brain Trp levels and thus produce positive effects on mood. Such positive effects on mood are only seen in healthy subjects following a 191-255\% increase in plasma TRP:LNAA levels. When plasma Trp levels are elevated beyond that range, mood in both healthy and vulnerable subjects is negatively affected. Given that Western and new world societies are increasingly demanding and food is generally abundantly available in these societies, insight in beneficial TRP:LNAA ratios in food for specific target groups may help the nutrition industry to adapt food to support our mood and cognition.

One group of individuals that is vulnerable to manipulations of the serotonergic system is the one that carries the $\mathrm{s}$ allele of the 5-HTTLPR polymorphism. Studies employing 5-HTT knockout mice and rats as a model have revealed that reduced 5-HTT expression (as hypothesised for 5-HTTLPR s-allele carriers) is associated with increased extracellular 5-HT levels, reduced intra-neuronal 5-HT levels and increased 5-HT synthesis. Also, various 5-HT receptors are down-regulated ${ }^{(32)}$. As to whether Trp levels or its transport over the blood-brain barrier is affected by the 5-HTTLPR genotype is unknown to date. Nonetheless, if 5-HT synthesis is enhanced to compensate lower 5-HT tissue levels, it may not be surprising that 5-HTTLPR s-allele carriers are more sensitive to Trp depletion ${ }^{(74)}$, an effect that has also been observed in 5-HTT knockout rats ${ }^{(87)}$. In contrast to ATD, ATS has a positive effect on mood, vigor and cortisol response after exposure to laboratory stress in ss subjects ${ }^{(108,109)}$. 5 -HTTLPR s-allele carriers are more sensitive to stress ${ }^{(133)}$ and are more prone to develop depression after adverse life events ${ }^{(31)}$. Given that ATS has been reported to dampen the negative effects on mood invoked by stress ${ }^{(108,109)}$, it is of interest to test whether (chronic) ATS improves stress coping and decreases risk for depression in s-allele carriers. Finally, to date, no studies on the effects of chronic Trp supplementation in human subjects have been conducted. With the recent discovery of powerful tools for enhancing available Trp through diet, such as hydrolysed protein or egg protein hydrolysate ${ }^{(8,9)}$, and promising data from ATS studies, chronic Trp supplementation studies in human subjects are warranted. Ultimately, enhancing available Trp in food such as cereals, maize and milk formulas may represent an efficient and cost-effective way of increasing mood and cognition, and thus our general
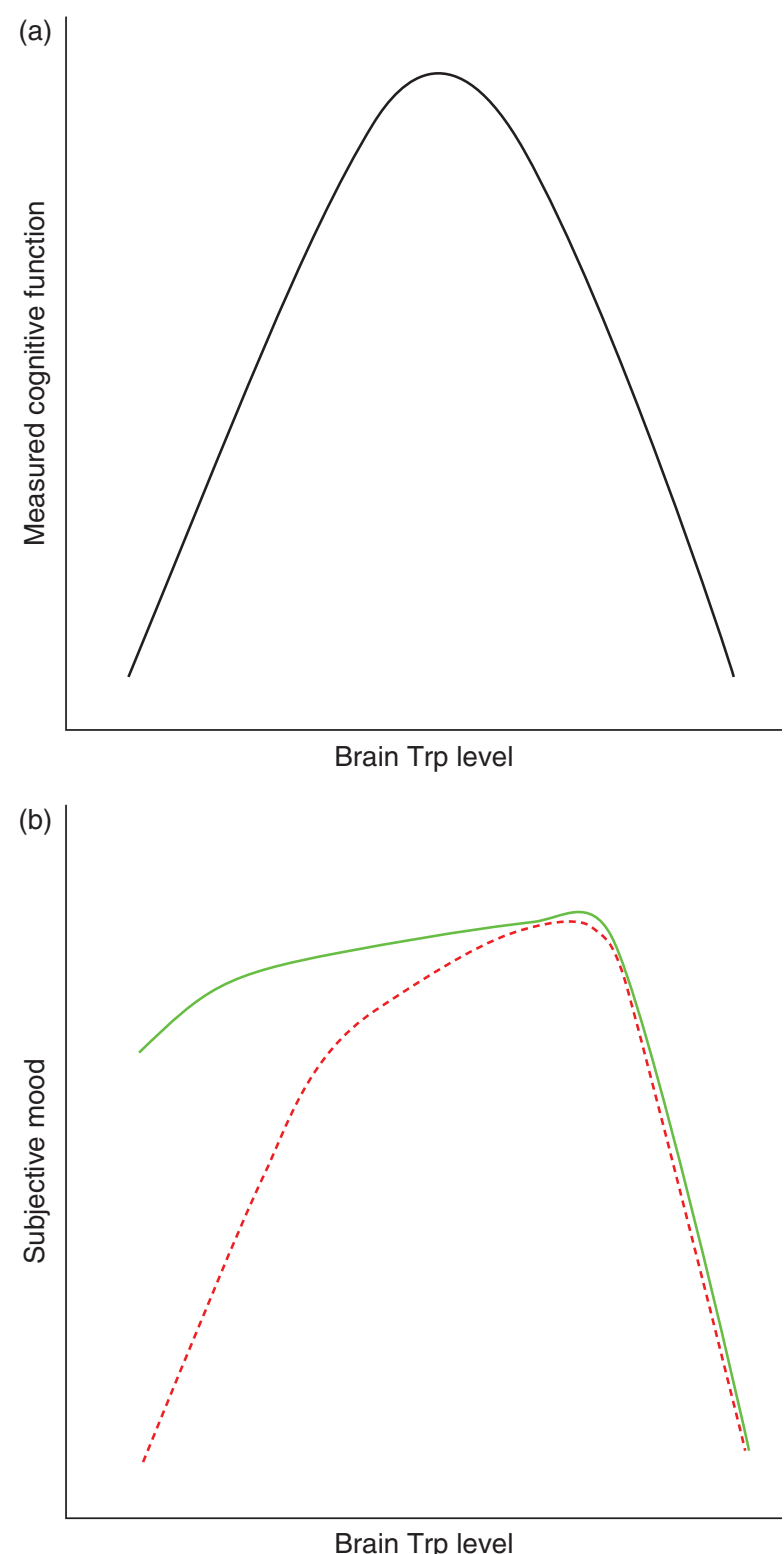

Fig. 1. Relationship between brain tryptophan (Trp) levels and cognition/ mood. In both healthy and vulnerable subjects too low and too high brain Trp levels result in impaired cognitive ability. This indicates that in the case of cognition, brain Trp levels should lie within an optimum range (a). The effects of small increases or decreases in brain Trp levels on the mood of healthy subjects is negligible $(b,-)$. Only large increases in brain Trp levels are able to improve mood significantly in these subjects. Conversely, in vulnerable subjects (b, -...) relatively small increases in brain Trp result in an improved mood. Unphysiologically high increases in brain Trp lead to negative effects on mood in both healthy and vulnerable subjects. (A colour version of this figure can be found online at http://www.journals.cambridge.org/nrr) 
well-being in the current increasingly demanding society. Specifically, insight into beneficial Trp:LNAA ratios in food for specific target groups - as presented here may help the nutrition industry to increase the functionality of our food. Specifically, contrary to popular belief, most common foods high in Trp do not increase the plasma TRP:LNAA ratio enough to exert any positive effects on mood/cognition, because they also contain large amounts of other LNAA. Hence, functional foods have to be designed containing much higher levels of Trp compared with other LNAA. With about $10 \%$ of the population over 12 years of age taking antidepressants in the USA alone $^{(134)}$ and the numerous side effects associated with their use, consumption of these functional foods to prevent depression in the first place is alluring for both clinicians and the general public.

\section{Acknowledgements}

J. R. H. is financially supported by The Netherlands Organisation for Scientific Research (NWO), grant no. 86410003. NWO had no further role in the design of the study, in the collection, analysis and interpretation of data, in the writing of the report, and in the decision to submit the paper for publication.

S. H., A. M., M. H. M. and J. R. H. wrote the manuscript, S. H. organised the references and made the figures, J. R. H. designed the manuscript, and J. R. H. and M. H. M. together wrote the conclusions.

There are no conflicts of interest.

\section{References}

1. American Psychiatric Association (1994) Diagnostic and Statistical Manual of Mental Disorders, 4th ed. Washington, DC: American Psychiatric Association.

2. Olivier B, Chan JS, Snoeren EM, et al. (2011) Differences in sexual behaviour in male and female rodents: role of serotonin. Curr Top Behav Neurosci 8, 15-36.

3. Wurtman RJ, Hefti F \& Melamed E (1980) Precursor control of neurotransmitter synthesis. Pharmacol Rev 32, 315-335.

4. Kerem Z, Lev-Yadun S, Gopher A, et al. (2007) Chickpea domestication in the Neolithic Levant through the nutritional perspective. J Archaeol Sci 34, 1289-1293.

5. Katz SH, Hediger ML \& Valleroy LA (1974) Traditional maize processing techniques in the new world. Science 184, $765-773$.

6. Morris CE \& Sands DC (2006) The breeder's dilemma yield or nutrition? Nature Biotechnol 24, 1078-1080.

7. Heine W, Radke M, Wutzke KD, et al. (1996) $\alpha$-Lactalbumin-enriched low-protein infant formulas: a comparison to breast milk feeding. Acta Paediatr 85, 1024-1028.

8. Mitchell ES, Slettenaar M, Quadt F, et al. (2011) Effect of hydrolysed egg protein on brain tryptophan availability. Br J Nutr 105, 611-617.

9. Markus CR, Firk C, Gerhardt C, et al. (2008) Effect of different tryptophan sources on amino acids availability to the brain and mood in healthy volunteers. Psychopharmacology 201, 107-114.
10. Silber BY \& Schmitt JAJ (2010) Effects of tryptophan loading on human cognition, mood, and sleep. Neurosci Biobehav Rev 34, 387-407.

11. Booij L, Van der Does AJ \& Riedel WJ (2003) Monoamine depletion in psychiatric and healthy populations: review. Mol Psychiatry 8, 951-973.

12. Mechan AO, Fowler A, Seifert N, et al. (2011) Monoamine reuptake inhibition and mood-enhancing potential of a specified oregano extract. Br J Nutr 105, 1150-1163.

13. Kroeze Y, Zhou H \& Homberg JR (2012) The genetics of selective serotonin reuptake inhibitors. Pharmacol Ther 136, 375-400.

14. Neal KB, Parry LJ \& Bornstein JC (2009) Strain-specific genetics, anatomy and function of enteric neural serotonergic pathways in inbred mice. J Physiol 587, 567-586.

15. Azmitia EC \& Whitaker-Azmitia PM (1995) Anatomy, cell biology and plasticity of the serotonergic system. In Psychopharmacology: The Fourth Generation of Progress, pp. 443-449 [FE Bloom and DJ Kupfer, editors]. New York: Raven Press.

16. Gutknecht L, Kriegebaum C, Waider J, et al. (2009) Spatiotemporal expression of tryptophan hydroxylase isoforms in murine and human brain: convergent data from Tph2 knockout mice. Eur Neuropsychopharmacol 19, 266-282.

17. Saura J, Andrés N, Andrade C, et al. (1997) Biphasic and region-specific MAO-B response to aging in normal human brain. Neurobiol Aging 18, 497-507.

18. Torres GE, Gainetdinov RR \& Caron MG (2003) Plasma membrane monoamine transporters: structure, regulation and function. Nat Rev Neurosci 4, 13-25.

19. Van Praag HM, Korf J \& Puite J (1970) 5-Hydroxyindoleacetic acid levels in the cerebrospinal fluid of depressive patients treated with probenecid. Nature 225, 1259-1260.

20. Malison RT, Price LH, Berman R, et al. (1998) Reduced brain serotonin transporter availability in major depression as measured by $\left[{ }^{123} \mathrm{I}\right]-2 \beta$-carbomethoxy- $3 \beta$-(4-iodophenyl)tropane and single photon emission computed tomography. Biol Psychiatry 44, 1090-1098.

21. Cowen PJ, Power AC, Ware CJ, et al. (1994) 5-HT1A receptor sensitivity in major depression. A neuroendocrine study with buspirone. BrJ Psychiatry 164, 372-379.

22. Sargent PA, Kjaer KH, Bench CJ, et al. (2000) Brain serotonin $1 \mathrm{~A}$ receptor binding measured by positron emission tomography with $\left[{ }^{11} \mathrm{C}\right] \mathrm{WAY}-100635$ : effects of depression and antidepressant treatment. Arch Gen Psychiatry 57, $174-180$.

23. Nutt DJ (2002) The neuropharmacology of serotonin and noradrenaline in depression. Int Clin Psychopharmacol 17, S1-S12.

24. Mawson AR \& Jacobs KW (1978) Corn consumption, tryptophan, and cross-national homicide rates. J Orthomol Psychiatry 7, 227-230.

25. Voracek M \& Tran US (2007) Dietary tryptophan intake and suicide rate in industrialized nations. J Affect Disord 98, 259-262.

26. Gordon JA \& Hen R (2004) The serotonergic system and anxiety. Neuromolecular Med 5, 27-40.

27. McDougle CJ, Epperson CN, Price LH, et al. (1998) Evidence for linkage disequilibrium between serotonin transporter protein gene (SLC6A4) and obsessive compulsive disorder. Mol Psychiatry 3, 270-273.

28. van Praag HM (2004) Can stress cause depression? Prog Neuropsychopharmacol Biol Psychiatry 28, 891-907.

29. Maes M \& Meltzer H (1995) The serotonin hypothesis of major depression. In Psychopharmacology: The Fourth Generation of Progress, pp. 933-944 [FE Bloom and DJ Kupfer, editors]. New York: Raven Press. 
30. Lesch K-P, Bengel D, Heils A, et al. (1996) Association of anxiety-related traits with a polymorphism in the serotonin transporter gene regulatory region. Science $\mathbf{2 7 4}$, $1527-1531$.

31. Caspi A, Sugden K, Moffitt TE, et al. (2003) Influence of life stress on depression: moderation by a polymorphism in the 5-HTT gene. Science 301, 386-389.

32. Kalueff AV, Olivier JD, Nonkes LJ, et al. (2010) Conserved role for the serotonin transporter gene in rat and mouse neurobehavioral endophenotypes. Neurosci Biobehav Rev 34, 373-386.

33. Sabol SZ, Hu S \& Hamer D (1998) A functional polymorphism in the monoamine oxidase A gene promoter. Hum Genet 103, 273-279.

34. Deckert J, Catalano M, Syagailo YV, et al. (1999) Excess of high activity monoamine oxidase A gene promoter alleles in female patients with panic disorder. Hum Mol Genet $\mathbf{8}$, 621-624.

35. Ni X, Sicard T, Bulgin N, et al. (2007) Monoamine oxidase A gene is associated with borderline personality disorder. Psychiatr Genet 17, 153-157.

36. Schulze TG, Müller DJ, Krauss H, et al. (2000) Association between a functional polymorphism in the monoamine oxidase A gene promoter and major depressive disorder. Am J Med Genet 96, 801-803.

37. Yu YW, Tsai SJ \& Hong CJ (2005) Association study of a monoamine oxidase A gene promoter polymorphism with major depressive disorder and antidepressant response. Neuropsychopharmacology 30, 1719-1723.

38. Domschke K, Braun M, Ohrmann P, et al. (2006) Association of the functional $-1019 \mathrm{C} / \mathrm{G}$ 5-HT1A polymorphism with prefrontal cortex and amygdala activation measured with $3 \mathrm{~T}$ fMRI in panic disorder. Int J Neuropsychopharmacol 9, 349-355.

39. Zhang X, Gainetdinov RR, Beaulieu J-M, et al. (2005) Loss-of-function mutation in tryptophan hydroxylase-2 identified in unipolar major depression. Neuron 45, 11-16.

40. Lin YM, Chao SC, Chen TM, et al. (2007) Association of functional polymorphisms of the human tryptophan hydroxylase 2 gene with risk for bipolar disorder in Han chinese. Arch Gen Psychiatry 64, 1015-1024.

41. Cichon S, Winge I, Mattheisen M, et al. (2008) Brainspecific tryptophan hydroxylase 2 (TPH2): a functional Pro206Ser substitution and variation in the 5'-region are associated with bipolar affective disorder. Hum Mol Genet 17, 87-97.

42. Ross DS, Fernstrom JD \& Wurtman RJ (1973) The role of dietary protein in generating daily rhythms in rat liver tryptophan pyrrolase and tyrosine transaminase. Metabolism 22, 1175-1184.

43. Yuwiler A, Oldendorf WH, Geller E, et al. (1977) Effect of albumin binding and amino acid competition on tryptophan uptake into brain. J Neurochem 28, 1015-1023.

44. Fernstrom JD, Larin F \& Wurtman RJ (1973) Correlation between brain tryptophan and plasma neutral amino acid levels following food consumption in rats. Life Sci 13, 517-524.

45. Fernstrom JD \& Wurtman RJ (1972) Brain serotonin content physiological regulation by plasma neutral amino acids. Science 178, 414-416.

46. Fernstrom JD \& Faller DV (1978) Neutral amino acids in the brain: changes in response to food ingestion. $J$ Neurochem 30, 1531-1538.

47. Carpenter LL, Anderson GM, Pelton GH, et al. (1998) Tryptophan depletion during continuous CSF sampling in healthy human subjects. Neuropsychopharmacology 19, $26-35$.
48. Nishizawa S, Benkelfat C, Young SN, et al. (1997) Differences between males and females in rates of serotonin synthesis in human brain. Proc Natl Acad Sci US A 94, 5308-5313.

49. Williams WA, Shoaf SE, Hommer D, et al. (1999) Effects of acute tryptophan depletion on plasma and cerebrospinal fluid tryptophan and 5-hydroxyindoleacetic acid in normal volunteers. J Neurochem 72, 1641-1647.

50. Ellenbogen MA, Young SN, Dean P, et al. (1996) Mood response to acute tryptophan depletion in healthy volunteers: sex differences and temporal stability. Neuropsychopharmacology 15, 465-474.

51. Smith KA, Clifford EM, Hockney RA, et al. (1997) Effect of tryptophan depletion on mood in male and female volunteers: a pilot study. Hum Psychopharmacol 12, 111-117.

52. Smith SE, Pihl RO, Young SN, et al. (1987) A test of possible cognitive and environmental influences on the mood lowering effect of tryptophan depletion in normal males. Psychopharmacology 91, 451-457.

53. Young SN, Smith SE, Pihl RO, et al. (1985) Tryptophan depletion causes a rapid lowering of mood in normal males. Psychopharmacology 87, 173-177.

54. Abbott FV, Etienne P, Franklin KBJ, et al. (1992) Acute tryptophan depletion blocks morphine analgesia in the cold-pressor test in humans. Psychopharmacology 108, 60-66.

55. Danjou P, Hamon M, Lacomblez L, et al. (1990) Psychomotor, subjective and neuroendocrine effects of acute tryptophan depletion in the healthy volunteer. Psychiat Psychobiol 5, 31-38.

56. Schmitt JA, Jorissen BL, Sobczak S, et al. (2000) Tryptophan depletion impairs memory consolidation but improves focussed attention in healthy young volunteers. $J$ Psychopharmacol 14, 21-29.

57. Scholes KE, Harrison BJ, O'Neill BV, et al. (2006) Acute serotonin and dopamine depletion improves attentional control: findings from the Stroop task. Neuropsychopharmacology 32, 1600-1610.

58. Harmer CJ, Rogers RD, Tunbridge E, et al. (2003) Tryptophan depletion decreases the recognition of fear in female volunteers. Psychopharmacology 167, 411-417.

59. Hayward G, Goodwin GM, Cowen PJ, et al. (2005) Lowdose tryptophan depletion in recovered depressed patients induces changes in cognitive processing without depressive symptoms. Biol Psychiatry 57, 517-524.

60. Van Der Veen FM, Evers EAT, Deutz NEP, et al. (2007) Effects of acute tryptophan depletion on mood and facial emotion perception related brain activation and performance in healthy women with and without a family history of depression. Neuropsychopharmacology 32, 216-224.

61. Cools R, Calder AJ, Lawrence AD, et al. (2005) Individual differences in threat sensitivity predict serotonergic modulation of amygdala response to fearful faces. Psychopharmacology 180, 670-679.

62. Riedel WJ, Klaassen T, Deutz NE, et al. (1999) Tryptophan depletion in normal volunteers produces selective impairment in memory consolidation. Psychopharmacology 141, 362-369.

63. McAllister-Williams R, Massey A \& Rugg M (2002) Effects of tryptophan depletion on brain potential correlates of episodic memory retrieval. Psychopharmacology 160, 434-442.

64. Benkelfat C, Ellenbogen MA, Dean P, et al. (1994) Mood-lowering effect of tryptophan depletion. Enhanced susceptibility in young men at genetic risk for major affective disorders. Arch Gen Psychiatry 51, 687-697.

65. Klaassen T, Riedel WJ, van Someren A, et al. (1999) Mood effects of 24-hour tryptophan depletion in healthy 
first-degree relatives of patients with affective disorders. Biol Psychiatry 46, 489-497.

66. Delgado PL, Charney DS, Price LH, et al. (1990) Serotonin function and the mechanism of antidepressant action. Reversal of antidepressant-induced remission by rapid depletion of plasma tryptophan. Arch Gen Psychiatry 47, 411-418.

67. Delgado PL, Price LH, Miller HL, et al. (1994) Serotonin and the neurobiology of depression. Effects of tryptophan depletion in drug-free depressed patients. Arch Gen Psychiatry 51, 865-874.

68. Moreno FA, Gelenberg AJ, Heninger GR, et al. (1999) Tryptophan depletion and depressive vulnerability. Biol Psychiatry 46, 498-505.

69. Smith KA, Fairburn CG \& Cowen PJ (1999) Symptomatic relapse in bulimia nervosa following acute tryptophan depletion. Arch Gen Psychiatry 56, 171-176.

70. Leyton M, Young SN, Blier P, et al. (1997) The effect of tryptophan depletion on mood in medication-free, former patients with major affective disorder. Neuropsychopharmacology 16, 294-297.

71. Booij L, Van der Does AJW, Haffmans PMJ, et al. (2005) The effects of high-dose and low-dose tryptophan depletion on mood and cognitive functions of remitted depressed patients. J Psychopharmacol 19, 267-275.

72. Merens W, Booij L, Haffmans PJ, et al. (2008) The effects of experimentally lowered serotonin function on emotional information processing and memory in remitted depressed patients. J Psychopharmacol 22, 653-662.

73. Neumeister A, Konstantinidis A, Stastny J, et al. (2002) Association between serotonin transporter gene promoter polymorphism (5HTTLPR) and behavioral responses to tryptophan depletion in healthy women with and without family history of depression. Arch Gen Psychiatry 59, 613-620.

74. Neumeister A, Hu XZ, Luckenbaugh DA, et al. (2006) Differential effects of 5-HTTLPR genotypes on the behavioral and neural responses to tryptophan depletion in patients with major depression and controls. Arch Gen Psychiatry 63, 978-986.

75. Walderhaug E, Magnusson A, Neumeister A, et al. (2007) Interactive effects of sex and 5-HTTLPR on mood and impulsivity during tryptophan depletion in healthy people. Biol Psychiatry 62, 593-599.

76. Walderhaug E, Herman AI, Magnusson A, et al. (2010) The short (S) allele of the serotonin transporter polymorphism and acute tryptophan depletion both increase impulsivity in men. Neurosci Lett 473, 208-211.

77. Firk C \& Markus CR (2009) Differential effects of 5-HTTLPR genotypes on mood, memory, and attention bias following acute tryptophan depletion and stress exposure. Psychopharmacology 203, 805-818.

78. Sambeth A, Blokland A, Harmer CJ, et al. (2007) Sex differences in the effect of acute tryptophan depletion on declarative episodic memory: a pooled analysis of nine studies. Neurosci Biobehav Rev 31, 516-529.

79. Roiser JP, Blackwell AD, Cools R, et al. (2006) Serotonin transporter polymorphism mediates vulnerability to loss of incentive motivation following acute tryptophan depletion. Neuropsychopharmacology 31, 2264-2272.

80. Porter RJ, Phipps AJ, Gallagher P, et al. (2005) Effects of acute tryptophan depletion on mood and cognitive functioning in older recovered depressed subjects. Am J Geriatr Psychiatry 13, 607-615.

81. Roiser JP, Muller U, Clark L, et al. (2007) The effects of acute tryptophan depletion and serotonin transporter polymorphism on emotional processing in memory and attention. Int J Neuropsychopharmacol 10, 449-461.
82. Franklin M, Bermudez I, Murck H, et al. (2012) Sub-chronic dietary tryptophan depletion - an animal model of depression with improved face and good construct validity. J Psychiatr Res 46, 239-247.

83. Izquierdo A, Carlos K, Ostrander S, et al. (2012) Impaired reward learning and intact motivation after serotonin depletion in rats. Behav Brain Res 233, 494-499.

84. Koot S, Zoratto F, Cassano T, et al. (2012) Compromised decision-making and increased gambling proneness following dietary serotonin depletion in rats. Neuropharmacology 62, 1640-1650.

85. Long AB, Kuhn CM \& Platt ML (2009) Serotonin shapes risky decision making in monkeys. Soc Cogn Affect Neurosci 4, 346-356.

86. Jenkins TA, Elliott JJ, Ardis TC, et al. (2010) Tryptophan depletion impairs object-recognition memory in the rat: reversal by risperidone. Behav Brain Res 208, 479-483.

87. Olivier JDA, Jans LAW, Korte-Bouws GAH, et al. (2008) Acute tryptophan depletion dose dependently impairs object memory in serotonin transporter knockout rats. Psychopharmacology 200, 243-254.

88. Nakamaru-Ogiso E, Miyamoto H, Hamada K, et al. (2012) Novel biochemical manipulation of brain serotonin reveals a role of serotonin in the circadian rhythm of sleep-wake cycles. Eur J Neurosci 35, 1762-1770.

89. Jans L, Korte-Bouws G, Korte S, et al. (2010) The effects of acute tryptophan depletion on affective behaviour and cognition in Brown Norway and Sprague Dawley rats. J Psychopharmacol 24, 605-614.

90. Fernstrom JD (1990) Aromatic amino acids and monoamine synthesis in the central nervous system: influence of the diet. J Nutr Biochem 1, 508-517.

91. Fernstrom JD \& Wurtman RJ (1971) Brain serotonin content: increase following ingestion of carbohydrate diet. Science 174, 1023-1025.

92. Yokogoshi H \& Wurtman RJ (1986) Meal composition and plasma amino acid ratios: effect of various proteins or carbohydrates, and of various protein concentrations. Metabolism 35, 837-842.

93. Markus CR, Olivier B, Panhuysen GE, et al. (2000) The bovine protein $\alpha$-lactalbumin increases the plasma ratio of tryptophan to the other large neutral amino acids, and in vulnerable subjects raises brain serotonin activity, reduces cortisol concentration, and improves mood under stress. Am J Clin Nutr 71, 1536-1544.

94. Orosco M, Rouch C, Beslot F, et al. (2004) $\alpha$-Lactalbuminenriched diets enhance serotonin release and induce anxiolytic and rewarding effects in the rat. Behav Brain Res $\mathbf{1 4 8}$, $1-10$.

95. Attenburrow MJ, Williams C, Odontiadis J, et al. (2003) Acute administration of nutritionally sourced tryptophan increases fear recognition. Psychopharmacology 169, 104-107.

96. Murphy SE, Longhitano C, Ayres RE, et al. (2006) Tryptophan supplementation induces a positive bias in the processing of emotional material in healthy female volunteers. Psychopharmacology 187, 121-130.

97. Markus CR, Panhuysen G, Tuiten A, et al. (1998) Does carbohydrate-rich, protein-poor food prevent a deterioration of mood and cognitive performance of stress-prone subjects when subjected to a stressful task? Appetite 31, 49-65.

98. Merens W, Booij L, Markus R, et al. (2005) The effects of a diet enriched with $\alpha$-lactalbumin on mood and cortisol response in unmedicated recovered depressed subjects and controls. Br J Nutr 94, 415-422.

99. Scrutton H, Carbonnier A, Cowen PJ, et al. (2007) Effects of $\alpha$-lactalbumin on emotional processing in healthy women. J Psychopharmacol 21, 519-524. 
100. Booij L, Merens W, Markus CR, et al. (2006) Diet rich in $\alpha$-lactalbumin improves memory in unmedicated recovered depressed patients and matched controls. J Psychopharmacol 20, 526-535.

101. Sobczak S, Honig A, Van Duinen MA, et al. (2002) Mood, prolactin and cortisol responses following intravenous L-tryptophan challenge: evidence for serotonergic vulnerability in first-degree relatives of bipolar patients. Int $J$ Neuropsychopharmacol 5, 249-254.

102. Sobczak S, Honig A, Schmitt JA, et al. (2003) Pronounced cognitive deficits following an intravenous L-tryptophan challenge in first-degree relatives of bipolar patients compared to healthy controls. Neuropsychopharmacology 28, 711-719.

103. Luciana M, Burgund ED, Berman M, et al. (2001) Effects of tryptophan loading on verbal, spatial and affective working memory functions in healthy adults. J Psychopharmacol 15, 219-230.

104. Morgan RM, Parry AMM, Arida RM, et al. (2007) Effects of elevated plasma tryptophan on brain activation associated with the Stroop task. Psychopharmacology 190, 383-389.

105. Cunliffe A, Obeid OA \& Powell-Tuck J (1998) A placebo controlled investigation of the effects of tryptophan or placebo on subjective and objective measures of fatigue. Eur J Clin Nutr 52, 425-430.

106. Sayegh R, Schiff I, Wurtman J, et al. (1995) The effect of a carbohydrate-rich beverage on mood, appetite, and cognitive function in women with premenstrual syndrome. Obstet Gynecol 86, 520-528.

107. Schmitt JAJ, Jorissen BL, Dye L, et al. (2005) Memory function in women with premenstrual complaints and the effect of serotonergic stimulation by acute administration of an $\alpha$-lactalbumin protein. J Psychopharmacol 19, 375-384.

108. Markus CR \& Firk C (2009) Differential effects of tri-allelic 5-HTTLPR polymorphisms in healthy subjects on mood and stress performance after tryptophan challenge. Neuropsychopharmacology 34, 2667-2674.

109. Cerit H, Jans LAW \& Van der Does W (2012) The effect of tryptophan on the cortisol response to social stress is modulated by the 5-HTTLPR genotype. Psychoneuroendocrinology 38, 201-208.

110. Markus CR, Verschoor E \& Smeets T (2012) Differential effect of the 5-HTT gene-linked polymorphic region on emotional eating during stress exposure following tryptophan challenge. $J$ Nutr Biochem 23, 410-416.

111. Markus CR, Panhuysen G, Jonkman LM, et al. (1999) Carbohydrate intake improves cognitive performance of stress-prone individuals under controllable laboratory stress. Br J Nutr 82, 457-467.

112. Markus CR, Olivier B \& de Haan EH (2002) Whey protein rich in $\alpha$-lactalbumin increases the ratio of plasma tryptophan to the sum of the other large neutral amino acids and improves cognitive performance in stress-vulnerable subjects. Am J Clin Nutr 75, 1051-1056.

113. Feurté S, Gerozissis K, Regnault A, et al. (2001) Plasma Trp/LNAA ratio increases during chronic ingestion of an $\alpha$-lactalbumin diet in rats. Nutr Neurosci 4, 413-418.

114. Stelt H, Broersen L, Olivier B, et al. (2004) Effects of dietary tryptophan variations on extracellular serotonin in the dorsal hippocampus of rats. Psychopharmacology 172, 137-144.

115. Collins CM, Kloek J \& Elliott JM (2013) Parallel changes in serotonin levels in brain and blood following acute administration of MDMA. J Psychopharmacol 27, 109-112.

116. Haider S, Khaliq S, Ahmed SP, et al. (2006) Long-term tryptophan administration enhances cognitive performance and increases $5 \mathrm{HT}$ metabolism in the hippocampus of female rats. Amino Acids 31, 421-425.
117. Haider S, Khaliq S \& Haleem DJ (2007) Enhanced serotonergic neurotransmission in the hippocampus following tryptophan administration improves learning acquisition and memory consolidation in rats. Pharmacol Rep 59, 53-57.

118. Coskun S, Ozer C, Gonul B, et al. (2006) The effect of repeated tryptophan administration on body weight, food intake, brain lipid peroxidation and serotonin immunoreactivity in mice. Mol Cell Biochem 286, 133-138.

119. Hickman AB, Klein DC \& Dyda F (1999) Melatonin biosynthesis. Mol Cell 3, 23-32.

120. Bombardelli E \& Morazzoni P (1995) Hypericum perforatum. Fitoterapia 66, 43-68.

121. do Rego J-C, Benkiki N, Chosson E, et al. (2007) Antidepressant-like effect of hyperfoliatin, a polyisoprenylated phloroglucinol derivative from Hypericum perfoliatum (Clusiaceae) is associated with an inhibition of neuronal monoamines uptake. Eur J Pharmacol 569, 197-203.

122. Sánchez-Mateo CC, Bonkanka CX \& Rabanal RM (2009) Hypericum grandifolium Choisy: a species native to Macaronesian region with antidepressant effect. J Ethnopharmacol 121, 297-303.

123. Skalisz LL, Beijamini V \& Andreatini R (2004) Effect of Hypericum perforatum on marble-burying by mice. Phytother Res 18, 399-402.

124. Viana A, do Rego J-C, von Poser G, et al. (2005) The antidepressant-like effect of Hypericum caprifoliatum Cham \& Schlecht (Guttiferae) on forced swimming test results from an inhibition of neuronal monoamine uptake. Neuropharmacology 49, 1042-1052.

125. Rodrigues AL, da Silva GL, Mateussi AS, et al. (2002) Involvement of monoaminergic system in the antidepressant-like effect of the hydroalcoholic extract of Siphocampylus verticillatus. Life Sci 70, 1347-1358.

126. Jin Z-L, Gao N, Zhou D, et al. (2012) The extracts of Fructus Akebiae, a preparation containing $90 \%$ of the active ingredient hederagenin: serotonin, norepinephrine and dopamine reuptake inhibitor. Pharmacol Biochem Behav 100, 431-439.

127. Knörle R (2012) Extracts of Sideritis scardica as triple monoamine reuptake inhibitors. J Neural Transm 119, 1477-1482.

128. Ofir R, Tamir S, Khatib S, et al. (2003) Inhibition of serotonin re-uptake by licorice constituents. J Mol Neurosci 20, $135-140$.

129. Dhingra D, Parle M \& Kulkarni SK (2004) Memory enhancing activity of Glycyrrhiza glabra in mice. J Ethnopharmacology 91, 361-365.

130. Dhingra D \& Sharma A (2006) Antidepressant-like activity of Glycyrrbiza glabra L. in mouse models of immobility tests. Prog Neuropsychopharmacol Biol Psychiatry 30, 449-454.

131. Mohajeri MH, Goralczyk R \& Dimpfel W (2012) Modulation of neurotransmission by a specified oregano extract alters brain electrical potentials indicative of antidepressant-like and neuroprotective activities. Neurosci Med 3, 37-46.

132. Bergström LS \& Lynöe N (2008) Enhancing concentration, mood and memory in healthy individuals: an empirical study of attitudes among general practitioners and the general population. Scand J Public Health 36, 532-537.

133. Owens M, Goodyer IM, Wilkinson P, et al. (2012) 5-HTTLPR and early childhood adversities moderate cognitive and emotional processing in adolescence. PLOS ONE 7, e48482.

134. Pratt LA, Brody DJ \& Gu Q (2011) Antidepressant use in persons aged 12 and over: United States, 2005-2008. In NCHS Data Brief, no. 76. Hyattsville, MD: National Center for Health Statistics. 